\title{
Alimentación normal del niño menor de 2 años. Recomendaciones de la Rama de Nutrición de la Sociedad Chilena de Pediatría 2013
}

\author{
CARLOS CASTILLO-DURÁN ${ }^{1}$, PAULINA BALBOA C. ${ }^{1}$, CLAUDIA TORREJÓN S. ${ }^{1}$, \\ KARLA BASCUÑÁN G. ${ }^{1}$, RICARDO UAUY D. ${ }^{1}$ \\ 1. Rama de Nutrición de Sociedad Chilena de Pediatría.
}

\begin{abstract}
Nutrition in childhood. Guides of the Nutrition Branch, Chilean Society of Pediatrics

Infant feeding habits are essential for child growth and development, as well as reducing various chronic disease risks; they can also alter later preferences of food. Obesity, allergies and other chronic diseases related to nutrition have become the most frequent problems in Chilean children. There is a lot of recent evidence, both international and domestic, about infant feeding habits, which forces an update to the 2004 Chilean guidelines. This study updates and proposes new feeding recommendations for the Chilean population during the first two years of life.

(Key words: Feeding, infants, guidelines, Chile).

Rev Chil Pediatr 2013; 84 (5): 565-572
\end{abstract}

\section{RESUMEN}

Los modos de alimentación adquiridos por el lactante son centrales para su crecimiento y desarrollo, así como para la disminución del riesgo de diversas enfermedades crónicas; pueden alterar además las preferencias alimentarias en otras edades. La obesidad, las alergias y otras enfermedades crónicas asociadas a la nutrición han pasado a ser los problemas más prevalentes en los niños chilenos. Hay abundante evidencia científica reciente tanto internacional como nacional acerca de los modos de alimentación del lactante, que están haciendo indispensable una actualización de las guías chilenas de alimentación del año 2004. Este artículo actualiza y propone nuevas recomendaciones de alimentación para población chilena durante los primeros dos años de vida. (Palabras clave: alimentación, lactantes, guías, Chile).

Rev Chil Pediatr 2013; 84 (5): 565-572

Recibido el 29 de agosto de 2013, aceptado para publicación el 28 de septiembre de 2013.

Este trabajo cumple con los requisitos sobre consentimiento /asentimiento informado, comité de ética, financiamiento, estudios animales y sobre la ausencia de conflictos de intereses según corresponda. 


\section{Introducción}

La Rama de Nutrición de la Sociedad Chilena de Pediatría elabora periódicamente recomendaciones en temas de su área de expertizaje; las últimas guías de alimentación para el niño menor de 2 años fueron publicadas y asumidas por el Ministerio de Salud el año 2004 y ahora se entrega una nueva actualización ${ }^{1}$.

La alimentación y nutrición normal del niño es un tema central en la edad pediátrica $\mathrm{y}$ en sus proyecciones para la vida adulta. El tema ha tenido un gran desarrollo a nivel de la investigación científica, que nos está permitiendo el perfeccionamiento de las guías chilenas de alimentación infantil.

Sigue siendo válido el criterio tradicional de catalogar una alimentación como normal si se asocia a un crecimiento y un desarrollo psicomotor normales, pero se han agregado otros importantes criterios: disminución del riesgo de enfermedades crónicas no transmisibles en edades posteriores (obesidad, diabetes, dislipidemias, hipertensión arterial (HTA), disminución del riesgo de enfermedad alérgica, regulación de los comportamientos asociados a la alimentación, regulación adecuada del ciclo sueño-vigilia, regulación de la actividad física, regulación de la inmunidad y riesgo de infecciones $^{2}$.

\section{Alimentación del lactante. Lactancia materna}

La lactancia materna (LM) es la alimentación central del lactante menor de 1 año. Esto incluye una lactancia materna exclusiva durante los primeros 6 meses de vida y con la inclusión de alimentación complementaria a partir del segundo semestre.

Actualmente hay evidencia suficiente que la LM en ambientes con nivel de desarrollo semejante o mejor que el de nuestro país, protege contra infecciones gastrointestinales $\mathrm{y}$ en menor grado infecciones respiratorias y que el efecto protector aumenta a mayor duración y exclusividad dentro del primer semestre de vida. Ésta también ha sido asociada a una disminución del riesgo de: muerte súbita, enfermedad atópica, adelanto en la aparición de enfermedad celíaca (en niños susceptibles), disminución del riesgo de enfermedades crónicas como la obesidad, la HTA, la diabetes mellitus tipo I, la enfermedad de Crohn y el linfoma. Algunos beneficios encontrados para la salud materna, incluyen la protección contra el cáncer de mama en mujeres premenopáusicas, cáncer de ovario y osteoporosis. Los principales puntos críticos relacionados con la lactancia materna en la actualidad son: la alimentación de la madre que está lactando; el momento de introducción de la alimentación complementaria y la lactancia materna en el segundo año de vida.

La evidencia actual ha descrito componentes de la alimentación de la madre que lacta en forma exclusiva y que pudieran tener influencia sobre el hijo, a saber: a) Variedad en la alimentación materna y adaptación del hijo en sabores e inmunología; b) Consumo materno de lípidos con ácidos grasos omega-3 y desarrollo del hijo; c) Inclusión-exclusión de ingredientes en alimentación materna y riesgo alérgico del hijo; d) Inclusión-exclusión de ingredientes en alimentación materna y riesgo de deficiencias nutricionales; e) Presencia de metales pesados en leche materna asociados a alimentación materna y posible riesgo de toxicidad en el hijo.

La mayor variabilidad posible en los ingredientes de la alimentación de la madre durante el embarazo y la lactancia parece estar asociada a una mejor aceptabilidad a nuevos ingredientes por el lactante y pudiera tener también asociación con un menor riesgo alérgico ${ }^{3}$.

Los ácidos grasos (AG) poliinsaturados de cadena larga (AGCL) metabólicamente más importantes para el desarrollo neurológico y visual (docosahexanoico, DHA, c22:6, n-3 y eicosapentaenoico, EPA, c20:5, n-3 y araquidónico, AA, c20:4, n-6) son derivados de los AG esenciales linoleico (18:2 n-6) (AL) y alfa-linolénico (18:3 n-3) (ALA). La fuente de ellos durante la vida fetal es la madre a través del transporte preferencial por la placenta y después de nacer, a través de la leche materna. Si bien, tanto el feto como el recién nacido pueden sintetizar DHA a partir de ALA, esta conversión es insuficiente para lograr los altos depósitos requeridos a nivel del sistema nervioso central (SNC). 
Las concentraciones de AA en la leche materna son constantes, mientras que las de EPADHA son variables y dependen de la ingesta materna. Un consenso internacional del año 2008, recomienda que mujeres embarazadas $\mathrm{y}$ en período de lactancia debieran ingerir al menos $200 \mathrm{mg} /$ día de DHA.

El paso de metales pesados y otros compuestos al niño a través de la leche materna es un tema actual de estudio por las posibles alteraciones en el corto y largo plazo; por su compromiso sobre la salud humana, los más estudiados han sido el Cadmio (Cd), el Plomo $(\mathrm{Pb})$ y el Mercurio $(\mathrm{Hg})$ por su posible efecto a nivel del SNC, causando neurotoxicidad ante dosis bajas pero prolongadas en un período crítico para el niño. Aún hay poca evidencia de que la exposición a través de la leche materna esté relacionada con algún tipo de daño; de hecho, existe alguna evidencia de que la LM puede contrarrestar algunos de los efectos negativos de la exposición a los contaminantes ambientales in útero. El cambiar la leche materna a las fórmulas tampoco garantiza una menor exposición a estos contaminantes ya que estudios han demostrado mayores cargas de metales pesados en lactantes alimentados con fórmula que en los alimentados con leche materna. La OMS considera un rango de concentración de 1,4- 1,7 ng Hg/g y 2-5 ng Pb/g como normal y seguro en la leche materna. Las organizaciones internacionales aconsejan evitar fumar durante la lactancia, ya que aumentaría la exposición a $\mathrm{Cd}$, el consumo de aguas contaminadas con $\mathrm{Hg}, \mathrm{Pb}$ y evitar el uso de pinturas que contengan $\mathrm{Pb}$, entre otras. En el caso del $\mathrm{Hg}$ cuya fuente principal de contaminación son algunos peces de mayor tamaño, la U.S. Food and Drug Administration (FDA) recomienda evitar el consumo de tiburón, pez espada, caballa o blanquillo, ya que pueden contener altas concentraciones de Hg. La recomendación de consumo propuesta corresponde a $340 \mathrm{~g}$ (2 porciones regulares) de una variedad de pescados y mariscos que sean bajos en $\mathrm{Hg}$ por semana, para cubrir los requerimientos de ácidos grasos de cadena larga n-3.

Por otro lado, en nuestra realidad nacional hay bajo riesgo de otras deficiencias nutricionales maternas y su efecto en el lactante, ex- cepto en hijos de madres vegetarianas estrictas, que presentan un mayor riesgo potencial de deficiencia de vitamina B12, zinc, hierro y ácidos grasos esenciales.

La importancia de la lactancia materna en el segundo año de vida sigue siendo poco estudiada en comunidades con mejor desarrollo socioeconómico. Seguimos recomendando su continuación mientras el niño mantenga un buen crecimiento, desarrolle hábitos saludables de alimentación y sea gratificante para la madre e hijo.

\section{Alimentación artificial del lactante. Fórmulas lácteas}

Ante la imposibilidad de continuar con la LM, la alimentación láctea artificial es una alternativa. En nuestro país, se dispone de fórmulas adaptadas comerciales y de la fórmula basada en leche de vaca entera en polvo entregada a través del Programa Nacional de Alimentación Complementaria (Purita fortificada).

La alternativa recomendada en este caso debiera ser una fórmula adaptada de inicio aportando $67 \mathrm{Kcal} / \mathrm{dL}$. Estas fórmulas cubren los requerimientos nutricionales de lactantes sanos, nacidos de término, durante los 12 primeros meses de vida. A su vez, existen en el mercado las fórmulas de continuación, las cuales debiesen ser utilizadas a partir de los 6 meses de vida. Las evidencias científicas apuntan a que esta diferenciación no resulta del todo indispensable. Tampoco está claramente demostrado que sea necesario continuar con este tipo de fórmulas más allá del primer año de vida, en comparación con dar simplemente leche de vaca semidescremada o descremada.

Las fórmulas comerciales enfatizan el contenido de otros componentes potencialmente requeridos por el lactante a estas edades. Dentro de ellos, los pro y prebióticos están presentes en forma natural en la leche materna y no hay evidencias suficientes a nivel clínico que ellos deban ser aportados en fórmulas comerciales para lactantes sanos. Los nucleótidos también son componentes de la leche materna, sin embargo la evidencia para 
su incorporación en las fórmulas artificiales es insuficiente.

Al año 2012 sigue estando disponible en Chile la Leche Purita Fortificada en el Programa Nacional de Alimentación Complementaria, la que se recomienda sea diluida al 7,5\% con adiciones de maltodextrina al 3\% (ingrediente que si no está disponible se podría reemplazar por sacarosa, a pesar de la recomendación de retrasar al máximo su incorporación hasta después de los 2 años) y aceite al 2\% para el primer semestre (soya o canola), o cereal $(5 \%)$ en vez de aceite desde el segundo semestre de vida hasta los 18 meses $(65-70 \mathrm{Kcal} /$ $\mathrm{dL}$ ), cuando es reemplazada por la Leche $\mathrm{Pu}-$ rita Cereal. Actualmente se discute en nuestro país su reemplazo por una fórmula adaptada con nombre genérico.

\section{Alimentación no láctea o alimentación complementaria}

La alimentación complementaria abarca no solamente alimentos sólidos o semisólidos (papillas o purés), sino que también alimentos líquidos (agua). Debemos tener en cuenta para nuestra realidad el aporte de energía necesario para cubrir los requerimientos del lactante vs la prevención del exceso de aporte de energía (obesidad) y de grasa total o de su composición (dislipidemias).

La introducción de alimentación complementaria se recomienda a partir de los 6 meses, tanto para aquellos lactantes que venían siendo alimentados al pecho exclusivo como para aquellos alimentados con fórmulas artificiales. No hay evidencias suficientes para establecer que el inicio de esta alimentación debe ocurrir solo a los 6 meses, o en un rango entre 5 y 6 meses de edad.

La razón más importante para la introducción de la alimentación complementaria es nutricional. En el transcurso del segundo semestre de vida, el niño alimentado al pecho puede manifestar signos de deficiencia de micronutrientes, en especial hierro y zinc, así como de energía y proteínas si continuara recibiendo LM en forma exclusiva. El inicio de la alimentación complementaria además depende de la madurez morfofuncional del niño: digestión y absorción adecuada de nutrientes, control de cabeza (capacidad de levantar y sostener la cabeza, en general a los 2 meses) y de tronco (capacidad de sentarse sin apoyo, en promedio a los 6 meses), uso de la musculatura masticatoria, erupción dentaria, incremento de las percepciones sensoriales (olfato, visión, gusto y tacto de alimentos), extinción del reflejo de extrusión y discriminación de nuevas texturas, sabores, olores, temperaturas y consistencia de los alimentos.

Por otra parte, el menor ya es capaz de manifestar sensaciones de hambre y saciedad, con aceptación o rechazo de los alimentos ofrecidos. A esta edad suelen aparecer las neofobias alimentarias manifestadas por una resistencia a la incorporación de alimentos nuevos. Esta característica es producto de un período madurativo de la conducta alimentaria y constituye un momento crucial en la adopción de patrones alimentarios adecuados; debe ofrecerse el alimento repetidamente hasta su aceptación normal.

La consistencia recomendada es la de una papilla o puré suave. Es importante que no contenga grumos, ni trozos de fibra que estimulen el reflejo de extrusión. Aproximadamente a los 8-9 meses de edad, etapa que generalmente coincide con la erupción de los incisivos superiores, aumenta la habilidad masticatoria, la lengua va desarrollando capacidad de movimientos laterales, por lo que la papilla puede tener la consistencia de puré molido, más consistente. Esta consistencia evoluciona progresivamente hasta que los alimentos son molidos con tenedor, cuando aparecen los primeros molares y la lengua puede movilizar adecuadamente el bolo de un lado a otro de la boca (12-18 meses de edad); sólo cuando erupcionan los molares se podrán ofrecer alimentos picados, asociado además a la capacidad del niño de tener movimientos coordinados laterales de la lengua (en general después de los 18 meses).

La alimentación complementaria debe aportar una densidad energética no inferior a la aportada a través de la leche materna. Su densidad energética en nuestro medio debiera estar entre 65 y $70 \mathrm{Kcal} / 100 \mathrm{~g}$. En su conjunto (2 papillas) no deben aportar más del $50 \%$ del aporte diario de energía (tabla 1). 
Tabla 1. Características de la alimentación complementaria para lactantes (6-12 meses)

\begin{tabular}{|c|c|c|c|c|c|c|c|c|}
\hline \multirow[b]{2}{*}{$\begin{array}{l}\text { Grupos de } \\
\text { alimentos }\end{array}$} & \multirow[b]{2}{*}{ Frecuencia } & \multirow[b]{2}{*}{ Alternativas } & \multicolumn{3}{|c|}{ Sopa puré 100 g, $67 \mathrm{Kcal}$} & \multicolumn{3}{|c|}{ Sopa puré 200 g, $135 \mathrm{Kcal}$} \\
\hline & & & $\begin{array}{l}\text { Cantidad } \\
\text { (g) }\end{array}$ & $\begin{array}{l}\mathrm{E} * \\
\text { (Kcal) }\end{array}$ & $\begin{array}{l}\text { Prot* } \\
(g)\end{array}$ & $\begin{array}{l}\text { Cantidad } \\
(\mathbf{g})\end{array}$ & 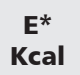 & $\begin{array}{l}\text { Prot* } \\
\text { (g) }\end{array}$ \\
\hline $\begin{array}{l}\text { Vegetales } \\
\text { verdes }\end{array}$ & Diaria & $\begin{array}{l}\text { Espinaca, zapallo italiano, } \\
\text { apio, lechuga, repollo, } \\
\text { porotos verdes, otras }\end{array}$ & 7,5 & 1,5 & 0,12 & 15 & 3 & 0,23 \\
\hline $\begin{array}{l}\text { Vegetales } \\
\text { coloreados }\end{array}$ & Diaria & $\begin{array}{l}\text { Zapallo, zanahoria, algas } \\
\text { marinas, champiñones, } \\
\text { betarraga, otras }\end{array}$ & 30 & 10 & 0,6 & 60 & 20 & 1,2 \\
\hline $\begin{array}{l}\text { Cereales y } \\
\text { tubérculos }\end{array}$ & Diaria & $\begin{array}{l}\text { Fideos, arroz, avena, papa, } \\
\text { quinoa, maíz }\end{array}$ & 15 & 16 & 0,3 & 30 & 32 & 0,6 \\
\hline Carnes & $3 \mathrm{v} / \mathrm{sem}$ & $\begin{array}{l}\text { Preferir: posta negra, rosada } \\
\text { ave, cerdo menos frecuente }\end{array}$ & 15 & 20 & 21 & 30 & (1) & \\
\hline $\begin{array}{l}\text { Pescados y } \\
\text { mariscos }\end{array}$ & $2 \mathrm{v} / \mathrm{sem}$ & $\begin{array}{l}\text { Merluza, reineta, jurel, } \\
\text { choritos, almejas, otros }\end{array}$ & 15 & 20 & 3,1 & 30 & 40 & 6,1 \\
\hline Legumbres & $2 \mathrm{v} / \mathrm{sem}$ & Porotos, lentejas, garbanzos & 35 & 27 & 4 & 70 & 53 & 8 \\
\hline Aceites & Diaria & Canola o soya & 2,5 & 22,5 & 0 & 5 & 45 & 0 \\
\hline $\begin{array}{l}\text { Puré de } \\
\text { frutas }\end{array}$ & Diaria & $\begin{array}{l}\text { Manzana, plátano, durazno, } \\
\text { kiwi, ciruela, melón, otras }\end{array}$ & 100 & 60 & 1 & & & \\
\hline
\end{tabular}

*Corresponde al promedio de energía y proteínas de las alternativas de alimentos propuestos. **El esquema de alimentación debe contemplar diariamente todos los grupos de alimentos de frecuencia diaria y 1 de frecuencia semanal en almuerzo y cena.

Al inicio de la alimentación complementaria la papilla o puré mixto se debe entregar en cantidades pequeñas pero crecientes, conforme avanza y acepta de buena forma la alimentación. Esta papilla debe contener cereales, vegetales variados y carnes bajas en grasa (porción de $30 \mathrm{~g}$ por comida, equivalentes a $1 \frac{1}{2}$ cajitas de fósforos), de preferencia de vacuno (posta negra o rosada), o bien pescado; se agrega aceite vegetal crudo (de preferencia de canola o soya) y postre de frutas crudas, molido y sin adición de azúcar. Eventualmente, se pueden agregar algunos condimentos naturales y de sabores no muy intensos como orégano, comino, laurel entre otros, siempre en cantidades pequeñas. En relación a la incorporación de cereales, se recomienda el consumo de aquellos que poseen gluten a partir de los 6 meses. No está claro si la introducción de gluten en el período entre 4 y 6 meses previene o retarda la aparición de la enfermedad celíaca en población general susceptible o en lactantes con antecedentes familiares directos de ella (de mayor riesgo).

Estimulando una progresión en la variedad de alimentos ofrecidos, se recomienda incor- porar las leguminosas guisadas con cereales entre los 7-8 meses. El pescado puede incorporarse entre lo 6-7 meses y al parecer este criterio también es válido para niños con antecedentes familiares directos de atopia o alergia alimentaria. El huevo puede incorporarse entre los 9-10 meses.

No se debe agregar sal a las comidas, ni azúcar a los postres. Los endulzantes artificiales (sacarina, aspartame, sucralosa, estevia) no debieran ser utilizados en los alimentos para niños menores de 2 años en forma directa ni en preparaciones o productos comerciales "light" o "diet".

El volumen de la alimentación complementaria debe ir aumentando en forma progresiva de acuerdo a la edad y aceptabilidad del lactante, comenzando con cantidades pequeñas (cucharaditas), hasta llegar a $150 \mathrm{~g} \mathrm{o} 3 / 4$ taza (medido en una taza de $200 \mathrm{~mL}$ ) a los 8 meses, más 100 g o $1 / 2$ taza de puré de fruta, aproximadamente. A partir de los 9 meses las cantidades adecuadas serán de 200 g o 1 taza de papilla dos veces al día (almuerzo y cena), manteniendo los $100 \mathrm{~g} \mathrm{o} 1 / 2$ taza de postre de fruta (tabla $1)$. 
Es importante mencionar que es en el período de los 6 a 24 meses donde se establecen la mayor parte de los hábitos, preferencias y aversiones alimentarias que condicionarán en gran medida el tipo de alimentación futura. Influyen varios factores de los que se pueden destacar: a) formación del gusto alimentario, asociado a varios factores genéticos y ambientales, en especial de los sabores dulce y salado; b) Transmisión genética: la respuesta de un niño frente a un alimento es adquirida, excepto por la preferencia innata por el dulce y la aversión al sabor amargo; c) Factores culturales: el entorno cultural puede reducir el número de alimentos que el niño pudiese incorporar en su alimentación (variedad de alimentos y grupos de alimentos), aumentar el número de comidas diarias y el tiempo entre comidas; d) Factores familiares: crear un ambiente alimentario positivo, físico (ej. TV apagada, pocos ruidos ambientales) y afectivo, es tan importante como aportar la cantidad adecuada de nutrientes. Un medio familiar con estas características estimulará a la adopción de hábitos alimentarios adecuados; e) Imitación: La observación de hábitos alimentarios de adultos cercanos al niño (padres, hermanos, abuelos, asesoras del hogar, personal de jardines infantiles) lo condicionará en mayor medida a comer lo que él ve comer.

\section{Alimentación durante el segundo año de vida}

Desde el año de edad el niño/a debe paulatinamente incorporarse a los hábitos y características de la alimentación familiar. Es aconsejable incorporar cuatro tiempos de comida principales durante el día, suspendiendo el horario nocturno de alimentación láctea (desayuno, almuerzo, once y cena), incorporándose a la comida propia del hogar, de consistencia molida y resguardando siempre que ésta sea saludable. En algunos ambientes además el niño recibe un $5^{\circ}$ tiempo de alimentación a media mañana (colación, merienda). Estas colaciones no son necesarias a esta edad; sin embargo, cuando la alimentación no permite respetar los horarios de alimentación (cada $4 \mathrm{~h}$ aproximadamente), el volumen y/o el aporte de nutrientes, o si está instaurado en el jardín o guardería infantil o escuela, podrá planificarse esta colación, pero adecuándolas a los requerimientos nutricionales del niño/a. Deben ser de características saludables, principalmente a base de frutas crudas, verduras, lácteos con bajo contenido graso, cereales y líquidos sin azúcar (tabla 2).

En nuestra realidad debiera educarse para retardar al máximo el inicio del consumo de golosinas, evitando en los primeros 2 años de vida jugos envasados o gaseosas, galletas de cualquier tipo, cereales azucarados, chocolates, confites o helados y productos salados, orientando a que las colaciones o meriendas que llevan los niños pre-escolares cuando asisten a un jardín infantil, sean de preferencia en base a frutas. Se recomienda evitar compra de golosinas por los padres y consumo por sus hijos, tomando en cuenta las modalidades de compra y consumo: compra impulsiva en centros comerciales, compra al paso, colaciones, fiestas o paseos infantiles, aporte por otros familiares (tabla 2).

\section{Suplementos nutricionales}

Recomendamos suplementar con vitamina D $400 \mathrm{U}$ diarias en todo los lactantes durante el primer año de vida; faltan estudios que pudieran justificar menores dosis en el norte del país con exposición regular al sol o mayores dosis en zonas australes del país; no hay evidencia suficiente aún para decidir una recomendación acerca de la magnitud de tiempo y condiciones para la exposición al sol del niño pequeño.

La suplementación con $\mathrm{Fe} 1 \mathrm{mg} / \mathrm{Kg} /$ día se justifica en lactantes alimentados en forma exclusiva al pecho desde los 4 meses y hasta el año; no se justifica en lactantes alimentados con fórmulas fortificadas. En lactantes prematuros se indica suplementar con $\mathrm{Fe} 2 \mathrm{mg} / \mathrm{Kg} /$ día desde el inicio del crecimiento acelerado; pudieran requerirse mayores dosis en lactantes con extremo bajo peso de nacimiento.

Se recomienda suplementar con zinc $3 \mathrm{mg} /$ día hasta los 12 meses de edad a lactantes prematuros $(<32$ semanas de gestación) o pequeños para la edad gestacional. 
Tabla 2. Frecuencia y cantidad de consumo de alimentos en el niño de 1-2 años

\begin{tabular}{|c|c|c|}
\hline Alimentos & Frecuencia & Cantidad sugerida \\
\hline Cereales $^{1}$ & Diaria & $\begin{array}{l}\text { Cereal precocido en la leche }\left(3,5 \text { medidas }^{2} \text { en } 250 \mathrm{ml}\right) \text {. Arroz, fideos, papas, otros, } \\
\text { almuerzo y cena ( } 1 / 2 \text { taza/vez) }\end{array}$ \\
\hline Vegetales & Diaria & $\begin{array}{l}\text { Verduras crudas o cocidas: zanahoria, acelga, zapallo, porotos verdes, otras; en almuerzo } \\
\text { y cena ( } 1 / 2 \text { taza/vez) }\end{array}$ \\
\hline Frutas & Diaria & $\begin{array}{l}\text { Frutas crudas como manzana, naranja, plátano, otras. Como postre o en colaciones } 2 \\
\text { veces al día ( } 1 \text { unidad) }\end{array}$ \\
\hline $\begin{array}{l}\text { Lácteos } \\
\text { Semidescremados }\end{array}$ & Diaria & $\begin{array}{l}\text { Lácteos semidescremados: leche, yogurt, quesos } 3 \text { veces/día. Ejemplo: desayuno, once y } \\
\text { colación }\end{array}$ \\
\hline $\begin{array}{l}\text { Carnes: vacuno, pollo, } \\
\text { pavo, cerdo }\end{array}$ & 2 veces/sem & Incluye aves, vacuno, cerdo, con poca grasa \\
\hline Pescado-mariscos & 2 veces/sem & Pescado y mariscos variados \\
\hline Legumbres & $1-2$ v./sem & $\begin{array}{l}\text { Varıar por trecuencla de consumo; } 40-40 \mathrm{~g} \text { almuerzo y cena Guisos/tortillas de verduras } \\
+ \text { huevo ( } 2-3 \text { semanales) }\end{array}$ \\
\hline Guiso verduras, huevo & $1-2 \mathrm{v} . / \mathrm{sem}$ & \\
\hline Aceites y otras grasas & Diaria & $\begin{array}{l}\text { De aceites vegetales, de preferencia sin cocción, } 2 \text { cucharaditas/día ( } 6 \mathrm{~mL} \text { ). La palta puede } \\
\text { incorporarse } 2-3 \text { veces/sem. ( } 1 \text { cucharada, al almuerzo o cena o con pan). Nueces, maní } \\
\text { después de los } 4 \text { años }\end{array}$ \\
\hline Azúcar y golosinas & & Evitar su consumo hasta después de los 2 años \\
\hline Agua & Diaria & Incluye todos los líquidos ingeridos, en promedio 4-5 tazas \\
\hline
\end{tabular}

1. Cereales incluyen el cereal instantáneo agregado a la leche al 3\%, o 1/2 porción de pan ( $1 / 4$ de marraqueta), al desayuno u once, 0 distribuido en ambas. 2. Definida la medida como aquella que hace $5 \mathrm{~g}$ de leche en polvo no instantánea.

En zonas del sur del país con bajo contenido de flúor del agua potable $(<0,3 \mathrm{ppm})$ se recomienda suplemento de $0,25 \mathrm{mg}$ diarios desde los 6 meses; asimismo, se recomienda postergar el uso de pastas dentales con flúor hasta los 6 años, dado que su ingestión al momento del aseo dental aumenta el riesgo de fluorosis.

En resumen, se entregan las nuevas Guías para la alimentación del niño menor de 2 años propuestas por la Rama de Nutrición de la Sociedad Chilena de Pediatría, con importantes modificaciones en relación a las comunicadas previamente el año 2004 y con una breve discusión sobre los fundamentos para cada una de ellas.

\section{Referencias}

1.- Torrejón C, Osorio J, Vildoso M, Castillo C: Alimentación del niño menor de dos años. Recomendaciones de la Rama de Nutrición de la Sociedad Chilena de Pediatría. Rev Chil Pediatr 2005; 76: 91-7. http://www. minsal.cl/ici/nutricion/Guia_Alimentacion.pdf

2.- Uauy RD, Castillo CD: Consecuencias de una nutrición infantil inadecuada para la salud y nutrición de la población. En: Nutrición y Alimentación del niño en los primeros años de vida. O’Donell A, Bengoa JM, Torún B, Caballero B, Lara Pantín E, Peña M. editores). Organización Panamericana de la Salud, OPS/OMS, Washington DC, 1997.

3.- Delgado-Noguera MF, Clavache JA, Bonfill Cosp X: Supplementation with long chain polyunsaturated fatty acids (LCPUFA) to breastfeeding mothers for improving child growth and development (Review) Cochrane Database Rev 2010; 8: CD007901.

4.- Koletzko B, Lien E, Agostoni C, et al: The roles of long-chain polyunsaturated fatty acids in pregnancy, lactation and infancy: review of current knowledge and consensus recommendations. J Perinat Med 2008; 36: 5-14.

5.- Joint FAO/WHO Expert Consultation on the Risks and Benefits of Fish Consumption Executive Summary 2529.

6.- Koyashiki G, Paoliello M, Tchounwou P: Lead levels in human milk and children's health risk: A systematic review. Rev Environ Health 2010; 25: 243-53.

7.- LaKind J, Wilkins A, Berlin C: Environmental chemicals in human milk: a review of levels, infant exposures and health, and guidance for future research. Toxicol Appl 
Pharmacol 2004; 198: 184-208.

8.- Cooke L, Fildes A: The impact of flavour exposure in utero and during milk feeding on food acceptance at weaning and beyond. Appetite 2011; 57: 808-11.

9.- Greer FR, Sicherer SH, Burks AW: Effects of early nutritional interventions on the development of atopic disease in infants and children: the role of maternal dietary restriction, breastfeeding, timing of introduction of complementary foods, and hydrolyzed formulas. Pediatrics 2008; 121: 183-91.

10.- Knip M, Virtanen SM, Kronberg-Kippilä K, et al: Sensitization at age 5 years age at the introduction of solid foods during the first year and allergic sensitization at age 5 years. Pediatrics 2010; 125; 50-9.

11.- Dewey KG, Cohen RJ, Rollins NC: WHO Technical background paper: Feeding of nonbreastfed children from 6 to 24 months of age in developing countries. Food Nutr Bull 2004; 25: 377-402.

12.- Cattaneo A, Williams C, Pallás-Alonso CR, et al: ESPGHAN's 2008 recommendation for early introduction of complementary foods: how good is the evidence? Matern Child Nutr 2011; 7: 335-43.

13.- Nwaru BI, Takkinen HM, Niemelä O, et al: Introduction of complementary foods in infancy and atopic sensitiza- tion at the age of 5 years: timing and food diversity in a Finnish birth cohort. Allergy 2013; 68: 507-16.

14.- Cohen R, Brown K, Canahuati J, Landa L, Dewey K: Effects of age of introduction of complementary foods on infant breast milk intake, total energy intake, and growth: a randomised intervention study in Honduras. Lancet 1994; 343: 288-93.

15.- Warddle J, Carnell S, Cooke L: Parental control over feeding and children's fruit and vegetable intake. How are they related? J Am Diet Assoc 2005; 105: 227-32.

16.- Niinikoski H, Lagström H, Jokinen E, et al: Impact of repeated dietary counseling between infancy and 14 years of age on dietary intakes and serum lipids and lipoproteins: the STRIP study. Circulation 2007; 116: 1032-40.

17.- Organización Mundial de la Salud (OMS): La alimentación del lactante y del niño pequeño: Capítulo Modelo para libros de texto dirigidos a estudiantes de medicina y otras ciencias de la salud. Biblioteca sede OPS, Washington DC 2010. ISBN: 978-92-75-33094-4 NLM WS120.

18.- Nwaru BI, Erkkola M, Ahonen S, et al: Age at the introduction of solid foods during the first year and allergic sensitization at age 5 years. Pediatrics 2010; 125: 50-9. 